\title{
Créer un flou référentiel : l'analyse de la structure pronom indéfini on $+V$ et de ses équivalents polonais sur l'exemple des textes fantastiques
}

\section{Creation of reference ambiguity/inaccuracy: the analysis of the structure indefinite pronoun $+V$ and its Polish equivalents based on fantasy texts}

\author{
Małgorzata Niziołek \\ Université Pédagogique de Cracovie \\ mniziolek1@gmail.com
}

\begin{abstract}
The starting point for the discussion is the statement that fantasy is born in the language. Among the linguistic measures that create the 'crack' in reality indefinite pronouns can be found. They make it possible to form the reference ambiguity/inaccuracy. Our aim is to describe linguistic mechanisms which conceal the perpetrator of action. This is why reflection on the structure indefinite pronoun $+\mathrm{V}$ and its Polish equivalents in the corpus of fantasy texts is offered. The purpose of the analysis is not to systematically describe the use of this structure. It is crucial to present the most important contexts in which the structure is present. The choice of corpus is a particularly important matter with regard to the obtained results. We would like to show that the structure indefinite pronoun on $+\mathrm{V}$ is one of the invariants of fantasy literature.
\end{abstract}

Keywords: the structure indefinite pronoun on $+\mathrm{V}$, traduction, invariant of fantasy literature

\section{REMARQUES PRÉLIMINAIRES}

Dans cet article, nous voulons proposer une réflexion autour de la construction pronom indéfini on $+V$ et de ses équivalents polonais dans le corpus des textes fantastiques, sans prétendre à une étude systématique de cette structure. L'équiva- 
lence que nous cherchons se situe au niveau sémantique. Muryn remarque à juste titre que « dans une analyse contrastive, surtout quand les phénomènes linguistiques que l'on compare proviennent de langues divergeant au niveau de leurs systèmes, le caractère universel du niveau sémantique le prédispose à jouer un rôle de tertium comparationis : un niveau auquel on peut se référer pour établir une équivalence entre des phénomènes comparés » (2019, à paraître).

Parmi les procédés langagiers qui créent « la fissure dans le réel » au détour des mots, nous pouvons classer les pronoms indéfinis. Ils mettent en effet en valeur une référence floue. Notre objectif est de décrire un des mécanismes linguistiques qui vise à occulter le sujet de l'action. Le fantastique émerge du choix des structures spécifiques et de leur enchaînement textuel. Comme le remarque Bellemin-Noël, l'« objet ou moment central de l'aventure, il est par définition indescriptible. [...] Néanmoins, le récit est contraint de le mettre en scène. Une rhétorique particulière se trouve alors mobilisée pour l'évoquer, le suggérer, imposer sa présence à travers les mots [...] L'auteur du fantastique doit les [=mots] obliger, durant un certain moment, à produire un non-encore-dit, à signifier un indésignable » $(1972$, p. 3). Dans cet article, nous allons nous pencher sur deux constructions différentes construites sur on $+V$ : la première, qui est résultative, et la seconde, qui contient un verbe médiatif. Les fonctions de ces constructions sont complètement différentes et leurs emplois semblent spécifiques pour des textes fantastiques dont l'un des objectifs est de faire naître l'incertitude.

\subsection{Corpus de travail}

Notre corpus est construit surtout de textes fantastiques français du XIX ${ }^{\mathrm{e}} \mathrm{s}$. et de leurs traductions polonaises et contient des textes de Guy de Maupassant, Villiers de L'Isle-Adam, Théphile Gautier et Prospère Mérimée. Les nouvelles fantastiques traduites en polonais viennent de l'anthologie des auteurs fantastiques du XIX ${ }^{\mathrm{e}}$ siècle intitulée " Fantastyczne opowieści » et contiennent des textes des auteurs suivants : Mérimée, Gautier, Maupassant, Balzac, France, Gautier (trad. Jachimecka, Krajewska, Rogowicz) et du recueil des nouvelles de Gautier « Stopa mumii i inne opowiadania fantastyczne » (trad. Parandowski, Dolatowska).

Cette analyse fait partie des recherches sur les exposants linguistiques de l'incertain dans des textes fantastiques en français du XIX ${ }^{\mathrm{e}}$ et du $\mathrm{XX}^{\mathrm{e}}$ siècles. Le corpus contient des textes d'auteurs français et d'auteurs étrangers traduits en français de l'anglais pour nous donner une vision contrastive du phénomène que nous considérons comme propre au genre fantastique, mais ayant des variantes se situant au niveau du discours (comme variantes du genre) qui s'expliquent par des différences extralinguistiques (peut-être psycho- ou socio- linguistiques). Dans notre article nous nous appuyons surtout sur des textes originaux français et sur leurs 
traductions polonaises. Cependant, dans le paragraphe 3.3 nous faisons aussi recours aux textes traduits en français (Lovecraft). Nous allons expliquer notre choix dans ce qui suit.

\subsection{Méthodologie du travail}

Notre analyse s'inscrit dans les travaux du groupe de recherches DiSem: sémantique, discours, inférence (Muryn, Niziołek, Hajok, Prażuch). Notre méthode d'analyse, onomasiologique, part du principe que chaque type de discours se caractérise par l'organisation de structures sémantiques complexes qui y dominent et, en même temps, par le choix de prédicats et d'arguments, la spécification de positions impliquées, etc. Ces structures se reconnaissent à travers leurs réalisations lexico-syntaxiques propres à un discours donné. Une structure sémantique complexe peut donc revêtir la forme d'une phrase complexe, d'une phrase simple, d'un SN, etc., ou bien être inférée en totalité ou en partie.

\section{QUELQUES PRÉCISIONS SUR LE PRONOM ON}

Le pronom on pose des problèmes d'identification aux grammairiens. Il est considéré comme un pronom personnel, un pronom indéfini voire comme pronom personnel indéfini (Sandfelf, 1970 ; Charaudeau, 1992 ; Grevisse et Goosse, 2002). « Il a ceci de particulier qu'il peut référer aussi bien à une personne précise qu'à un générique, à un collectif clairement identifiable qu'à un groupe de personnes à l'étendue et aux limites vagues » (Landragin et Tanguy, 2014, p. 99). Certains le classent parmi les mots grammaticaux à cause de sa déperdition de matière au niveau lexical, les autres le rangent parmi des mots lexicaux (notions générales qu'ils contiennent les rapprochent sémantiquement des noms) (Rémi-Giraud, 2010, p. 53-58). Il peut jouer un double rôle : celui d'un pronom personnel (quand il réfère à je, tu, il, nous, vous, ils), d'un pronom indéfini (quand il renvoie à quelqu'un, des gens). On est en quelque sorte la forme de référence qui correspond le mieux à la notion de «groupe flou » (Landragin et Tanguy, 2014). L'interprétation de on dépend du contexte dans lequel il apparait. Comme on s'interprète de multiples manières, cet article portera uniquement sur des emplois qui, d'après nous, peuvent être considérés comme propres aux textes fantastiques et permettent au fantastique de prendre forme. Et le fantastique n'existe pas en dehors du texte.

Comme nous considérons la construction on $+V$ comme l'un des mécanismes qui fait naître l'hésitation ${ }^{1}$ dans le fantastique, il est intéressant d'observer comment

\footnotetext{
${ }^{1}$ D'après Todorov (1970), l'hésitation est au cœur du fantastique. Bouvet (2007), à son tour, met l'accent sur la notion d'indétermination dans des textes fantastiques.
} 
cette construction est rendue dans des textes traduits en polonais et quel en est la forme. Notre hypothèse est que s'il s'agit d'un élément générique, il devrait être mis au premier plan dans des textes traduits (ce qui n'est pas le cas des mots/structures qui ne constituent que la massa tabulettae des textes fantastiques).

\section{L'ANALYSE DE LA CONSTRUCTION $O N+V$}

Nous voulons nous concentrer sur deux emplois de la construction on $+V$. Dans le premier, on $+V$ fonctionne comme une construction résultative, et le deuxième emploi, où la construction on $+V$ réfère à un acte de parole dans lequel les verbes dire/parler/raconter transmettent l'idée de parole répétée qui acquiert le sens de « bruit qui court », de « rumeur ».

\subsection{Construction résultative}

Tout au début de cet article, nous avons écrit que cette analyse porterait sur le corpus de textes fantastiques. Cependant, nous allons commencer par seulement une nouvelle de Guy de Maupassant, intitulée le Horla. Dans ce texte, nous avons repéré un emploi très intéressant de la structure $o n+V$. L'accumulation de ladite structure a permis de créer un effet que nous allons décrire dans ce qui suit. L'importance de cette construction et des problèmes traductologiques qu'elle produit ont été abordés par Lécrivain (1991). Comme le remarque Lécrivain, par rapport à d'autres nouvelles de Maupassant, c'est la seule où le phénomène (un événement/état/agent étrange) n'est pas nommé directement. Déjà le titre nous suggère l'impossibilité de dire le phénomène ${ }^{2}$.

Les phrases avec construction on $+V$, hors contexte, ne pourraient pas produire le même effet. La signification des phrases (1-7), et surtout la fonction du pronom on, est le résultat de la synergie qui existe entre tous les éléments. La reprise de la structure on $+V$ constitue une étape cruciale pour la construction de l'effet fantastique et assure en même temps la progression du texte. D'où l'importance pour le traducteur de transmettre cette information lors de la traduction :

(1) Tout à coup, il me sembla que j'étais suivi, qu'on marchait sur mes talons, tout près, à me toucher.

(2) On n'a touché à rien.

(3) On a bu.

\footnotetext{
${ }^{2}$ Le titre « Le Horla » nous informe qu'il s'agit d'un être hors là, un signe de l'être. Il est de par sa nature innommable. D'ailleurs, dans le texte, le phénomène n'est jamais nommé de façon directe : cet inconnaissable, il, Celui, l'Etre.
} 
(4) On avait donc bu cette eau?

(5) On a encore bu toute ma carafe cette nuit.

(6) On n'a touché ni au vin, ni au pain, ni aux fraises.

(7) On avait bu toute l'eau! On avait bu tout le lait.

Lécrivain souligne qu' « on est une sorte de non-information, de marque zéro qui ne porte aucune marque spécifique de personne et recouvre une troisième personne sans genre et sans nombre et [...] ce pronom peut éventuellement se substituer aux pronoms personnels de la première et de la deuxième personne. Ce pronom absorbe des contraires et des oppositions et introduit par conséquent une nette ambiguïté, multipliant les possibilités de signifiés » (1991, p. 137). Dans le cas des phrases analysées, la référence de on n'est pas ambiguë, elle est sous-déterminée. Même si le personnage voudrait inclure son je dans on pour pouvoir expliquer ce qui se passe (ce qu'il essaie de faire à plusieurs reprises : On a encore bu toute ma carafe cette nuit ; - ou plutôt, je l'ai bue! Mais, est-ce moi ? Est-ce moi ? Qui serait-ce? Qui ? Oh! mon Dieu! Je deviens fou!), tous les événements invalident cette explication. Lui-même ne croit pas à une explication rationnelle. On ne porte aucune marque spécifique de personne, d'où il devient particulièrement prédestiné à créer l'effet fantastique. Il est en même temps présence et absence.

Les exemples de (1) à (7) ont été condensés dans la nouvelle. D'autres exemples, avec la même fonction, mais plus dispersés, apparaissent aussi dans notre corpus.

(8) Alors seulement je compris toute l'horreur de ma position. On m'emporta; le roulement sourd des roues m'apprit que j'étais dans le corbillard (Gautier, Onuphrius).

(9) Ensuite on avait redescendu l'escalier. La porte de Mme de Peyrehorade s'était fermée (Mérimée, Vénus d'Ille).

Dans les exemples (8) et (9), de nouveau, le narrateur ne précise pas la référence de $o$. Tout conduit dans ces fragments à augmenter l'indétermination : une question rompue, des adjectifs : confuse, obscure, énigmatique, construction impersonnelle : il se passait.

Les phrases citées ont une propriété fonctionnelle commune : « de la relation prédicative, qui est constituée par deux termes, un thème et un rhème (propos), elles n'explicitent que le rhème. Ce sont des énoncés qui ont une autonomie grammaticale (structurelle), mais qui manquent d'autonomie sémantique (communicative)» (Karolak, 2000, p. 101-102). Ce sont des constructions résultatives. Elles mettent l'accent sur le résultat et n'explicitent pas l'agent. Les deux membres de phrase, le nom-sujet et le verbe-prédicat grammatical, constituent le rhème, qui seul se trouve dans le champ de l'assertion. « Ce sont donc des phrases athématiques et, par ce fait 
même, contextuelles pour lesquelles le thème doit être cherché ailleurs, c'est-à-dire dans l'environnement contextuel (linguistique) ou situationnel (pragmatique)» (Karolak, 2000, p. 105). Le thème est contenu dans le temps - à ce moment-là. Les constructions on $+V$ que nous venons de décrire servent à bloquer la cause, donc en même temps l'agent. Elles sont aspectuellement dérivées de la structure causative complète, mais l'explicitation de la cause étant bloquée, seul le résultat apparaît. Ces structures saisissent le résultat : il n'y a plus d'eau (carafe vide), il n'y a plus de lait, la porcelaine est tracassée, la porte est une fois ouverte, une fois fermée, etc. On n'explicite pas l'agent de l'action, on annonce seulement qu'il a fait quelque chose. Dans l'analyse aspectuelle, il s'agit d'une structure causativerésultative qui se compose de deux constantes : la première, que nous identifions comme cause, quelque chose qui s'est passé ou comme quelqu'un a fait quelque chose et la seconde qui réfère à un nouvel état - effet du changement (un nouvel état est advenu). Cette structure présuppose aussi qu'un état précédent a cessé d'exister au profit du nouveau. La fin de l'état précédent et le moment initial d'un nouvel état ont le même point de référence : celui de la cause. Le deuxième segment est identifié comme inchoatif, il est perfectif puisqu'il est borné par le moment initial d'un nouvel état, mais il bloque la spécification de la cause. Cette structure peut avoir plusieurs réalisations linguistiques, il se peut que la construction porte sur la cause en laissant le résultat inféré, comme dans le cas, entre autres, du perfectum (passé composé) qui se concentre sur l'explicitation de la cause en laissant l'effet à inférer (comme dans les exemples précédents). Le point de référence, qui est, comme nous venons de le dire, identique pour la cause, pour la fin de l'ancien état et pour le moment initial du nouvel état, peut émerger dans le texte de différentes manières, par exemple à l'aide d'adverbiaux temporels, aspectuels, etc. On le voit bien dans le corpus des textes fantastiques où la construction en question coopère avec des adverbes temporels ponctuels qui insistent sur le surgissement: brusquement, soudain, tout à coup, subitement (Muryn, 2010). L'adverbe de temps tout à coup, ou tout autre de sens voisin, renforce le contraste et fait d'une action qui s'insère au premier plan une action qui s'y propulse pour y «éclater » (Weinrich, 1989, p. 449). Ces adverbes démarquent des phrases avec la construction on $+V$ de l'état résultant. Ils attirent l'attention sur la soudaineté de la situation et soulignent un changement.

Les exemples cités (sauf 1 et 8) se prêtent à la transformation passive parce que les verbes inclus dans les constructions sont transitifs :

L'eau avait été bue. / Rien n'a été touché. / Le vin n'a été touché, etc.

Elle permet de changer l'ordre phrastique de façon à effacer l'agent de l'action, de ne pas l'expliciter. La construction passive obtient alors une valeur résultative en se concentrant sur l'effet obtenu d'objet qu'elle thématise. On voit bien la différence entre les résultats obtenus par les deux réalisations : la première, impersonnelle porte 
sur l'événement, la cause, tandis que la construction passive ne communique que l'état en ôtant une partie de dynamisme au récit se situant à « l'arrière-plan ». Même si sémantiquement les phrases avec on $+V$ et leurs transformations passives se ressemblent (elles dissimulent l'agent), elles présentent une autre vision des choses. Avec on $+V$ on communique qu'il y a un agent qu'on ne connait pas. Toute la phrase est rhématique. La construction passive focalise sur le manque d'eau, de vin etc. qui constitue le thème et ne s'intéresse pas à l'agent.

Dans tous les exemples présentés supra, on peut remplacer on par quelqu'un. Mais par rapport à on, le pronom personnel quelqu'un renvoie à un humain, et la sous-détermination créée par on disparaît :

\section{Quelqu'un a frappé / Quelqu'un a marché.}

3.2. Traduction de la construction $o n+V$ par les constructions impersonnelles en - no $/$ - to

Pour ce qui est de la traduction de la structure en question, elle est souvent reprise à l'aide des constructions impersonnelles en -no / -to, issues diachroniquement des formes neutres du participe passif, fonctionnant en synchronie comme des formes verbales finies employées dans une construction active (Krasnowolski, 1909, p. 24 ; Netteberg, 1953, p. 132 ; Wolińska, 1978, p. 65). Grzegorczykowa souligne que certains verbes, même s'ils peuvent être conjugués à toutes les personnes, possèdent une forme impersonnelle qui permet non seulement de ne pas expliciter l'agent de l'action, mais de l'exclure de cette possibilité. Ce sont des formes en -no / -to, qui, contrairement aux formes avec się pronominal (pisze się / napisało się, przynosi się / przyniosto się) se rapportent aux situations où les faits mentionnés sont indépendants du savoir et de la conscience du locuteur (2008, p. 106). Ces constructions peuvent être formées à partir des verbes tant imperfectifs que perfectifs exprimant les actions (« słowa czynności ludzkiej», comme le dit Krasnowolski $(1909, \S 26))$ ou les états humains. Dans ces constructions sans sujet, la place du sujet est bloquée et occupée par un sujet zéro avec une contrainte sémantique + hum / anim (Miladi, 2012, p. 59-63) . $^{3}$ À vrai dire, la restitution du sujet se fait dans ces constructions à travers les implications sémantiques du prédicat central, mais, comme elles n'explicitent aucun élément de l'ensemble d'arguments impliqués, elles sont ouvertes à d'autres choix, si, bien sûr, le contexte autorise une nouvelle interprétation. Il semble qu'elles servent d'équivalents les plus proches aux constructions on $+V$, puisqu'elles ont les mêmes caractéristiques aspectuelles et fonctionnelles

\footnotetext{
${ }^{3}$ Pour plus d'informations sur le fonctionnement de ces structures, nous renvoyons au mémoire de synthèse de Miladi (2012).
} 
(thème-rhème). Dans le corpus analysé, on retrouve plusieurs exemples avec des constructions impersonnelles en -no /-to :

(10) Styszałem szelest sukni, szmer trzewików, szepty, sttumione śmiechy, hałas odsuwanych foteli i stotu. Rozstawiano porcelanę, otwierano i zamykano drzwi; działo się coś niezwyklego. (Gautier, Klub haszyszystów, trad. Parandowski)

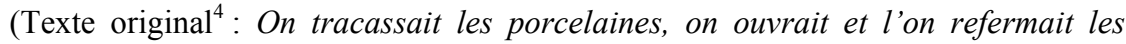
portes ; il se passait quelque chose d'inaccoutumé. Un personnage énigmatique m'apparut soudainement.)

(11) Spałem bardzo dobrze, nie śniqc ani o niedźwiedziach, ani o złodziejach. Rano kończyłem właśnie moja toaletę, kiedy zapukano do drzwi. (Mérimée, Lokis, trad. Jachimecka)

(TO : Je dormis fort bien, sans rêver d'ours ni de voleurs. Le matin, j'achevais ma toilette, quand on frappa à ma porte.)

(12) Hrabia pochylit się nad czara, której woda natychmiast zmąciła się pod jego spojrzeniem i przybrała odcień opalowy, jakby wlano do niej krople jakiejś esencji; tęczowy krąg barw otoczyt brzeg naczynia, ujmując w ramy obraz, który się już wylaniat spoza białawej chmury. (Gautier, Avatar, trad. Parandowski)

(TO : Le comte s'inclina sur la coupe, dont l'eau se troubla bientôt sous son regard et prit des teintes opalines, comme si l'on y eût versé une goutte d'essence; un cercle irisé des couleurs du prisme couronna les bords du vase, encadrant le tableau qui s'ébauchait déjà sous le nuage blanchâtre. Le brouillard se dissipa.)

Seulement deux exemples tirés de «Le Horla» ont été rendus par des constructions équivalentes en polonais qui terminent par -no / -to. Dans les exemples ci-dessous on produit le même effet que dans la traduction :

(13) Nie ruszono niczego. / On n'a touché à rien.

(14) Wypito. / On a bu.

D'autres phrases sont reprises soit par des phrases passives qui, comme nous l'avons déjà signalé, informent seulement du résultat :

(15) Więc woda została wypita!... / On avait donc bu cette eau ? / trad. L'eau avait été bue.

(16) Więc woda została wypita! Była [karafka / la carafe]) próżna!... była kompletnie próżna! / On a encore bu toute ma carafe cette nuit.

(17) Wszystka woda byta wypita! Wszystko mleko także!... Boże!... I On avait bu toute l'eau! On avait bu tout le lait!

(18) Wino, chleb i poziomki pozostały nietknięte. / On n'a touché ni au vin, ni au pain, ni aux fraises.

\footnotetext{
${ }^{4}$ Dorénavant TO.
} 
Soit on est remplacé par le pronom indéfini ktoś ('quelqu'un') :

(19) Ktoś wypit znowu cała karafkę tej nocy. / On a encore bu toute ma carafe cette nuit.

(20) Naraz wydało mi się, że za mna ktoś idzie, że mi następuje na pięty, dotyka mnie, że jest tuż-tuż... / Tout à coup, il me sembla que j'étais suivi, qu'on marchait sur mes talons, tout près, à me toucher.

Même si la traduction permet de maintenir le doute sur l'identité du phénomène, le remplacement de on par l'équivalent polonais de quelqu'un (ktoś) réduit le flou référentiel. On renvoie aussi bien aux humains qu'aux animés (en fonction du verbe employé) tandis que quelqu'un réfère uniquement aux humains. Dans le cas des textes fantastiques, il s'agit de construire le maximum de doute et d'incertitude. Quelqu'un, même si classé comme indéfini, est moins indéterminé par rapport à on. On dirait que le texte, en renvoyant directement à l'humain, falsifie / trahit et contredit le texte original en lui ôtant la valeur de l'incertain. Dans d'autres traductions, on retrouve également le recours au pronom ktoś :

(21) Ustyszatem chrobot kótek przesuwających się po pręcie, jakby ktoś szybko rozsuwat zastony przy lóżku. Obudzilem się; w każdym razie we śnie zdawało mi się, że się budzę. Nie zobaczylem nikogo. (Gautier, Omfala, trad. Krystyna Dolatowka, 1980) (TO : J'entendis les anneaux des rideaux de mon lit glisser en criant sur leurs tringles, comme si l'on eût tiré précipitamment les courtines. Je m'éveillai ; du moins dans mon rêve il me sembla que je m'éveillais. Je ne vis personie.)

\subsection{La construction on $+V$ et valeur évidentielle}

L'extraction automatique de $o n+V$ du corpus ${ }^{5}$ plus large que celui qui constitue la base de cet article nous a permis de découvrir encore un emploi de cette construction : on + dire (dit / disait). Même si elle apparait surtout dans des textes traduits en français (nouvelles de Lovecraft), nous avons décidé d'en donner quelques exemples, vu la fonction discursive qu'elle assume. La spécificité de cet emploi consiste à transmettre une information dont la valeur est traditionnellement décrite en termes de " rumeur », de " bruit qui court», ou de " ouï-dire ». Les énoncés introduits par on dit / disait sont considérés comme des marqueurs évidentiels. On les définira d'après Dendale et Tasmowski comme étant " une expression langagière qui apparaît dans l'énoncé et qui indique si l'information transmise dans cet énoncé a été empruntée par le locuteur à autrui ou si elle a été créée par le locuteur lui-même, moyennant une inférence ou une perception » (1994,

\footnotetext{
${ }^{5}$ Le corpus des textes fantastiques en français du XIX ${ }^{\mathrm{e}}$ et du début du $\mathrm{XX}^{\mathrm{e}}$ siècles. L'extraction est faite avec le logiciel TXM.
} 
p. 5). Pourquoi alors marquer la source d'un savoir? «En signalant dans l'énoncé la façon dont il a obtenu l'information qui y est transmise, le locuteur offre à son interlocuteur la possibilité d'évaluer lui-même la fiabilité de cette information " (Kim, 2004, p. 42). Les énoncés introduits par ces formes renvoient à la transmission d'une information à un public, et indiquent qu'il s'agit nécessairement d'une information de seconde main. Le locuteur se dissocie de l'information qu'il transmet. Le locuteur d'un énoncé, on dit / disait ne peut, en effet, avoir été le témoin direct de l'événement qu'il relate, d'où le fait que ce locuteur soit posé comme ne garantissant pas la véracité de l'information qu'il transmet. Ces formes marquent la dilution linguistique de la responsabilité du locuteur et mettent l'accent sur le non-engagement de l'énonciateur à l'égard des faits qu'il présente (Thuillier, 2004, p. 30). Les analyses effectuées nous permettent de faire une hypothèse que le choix de ces formes semble approprié au fantastique et y véhicule une information de type générique. Thuillier résume ainsi le mécanisme du fonctionnement de constructions porteuses de "ouï-dire »: «on a affaire, dans ces énoncés, à un événement en lui-même inaccessible (que l'on note donc $\mathrm{X}$ ), mais qui se trouve appréhendé grâce à un récit de cet événement (c'est-à-dire par le biais d'une entité seconde par rapport à $X$, notée $X^{\prime}$ ). On retrouve donc un $X$ qui reste hors d'atteinte, mais qui se trouve appréhendé par $\mathrm{S}$ par le biais d'un $\mathrm{X}$ ', second par rapport à $\mathrm{X}$ » (2004, p. 30). Les exemples de (22) à (24) illustrent l'emploi en question :

(22) On disait même qu'il avait traité avec le grand-prêtre, celui qu'on ne doit pas décrire, qui porte un masque de soie jaune et vit seul dans un monastère préhistorique. (Lovecraft, La quête onirique de Kadath inconnue)

(23) J'avais alors huit ans, et l'on disait qu'elle était morte de chagrin après le suicide de mon oncle Douglas, son fils aîné. (Lovecraft, Cauchemar d'Innsmouth)

(24) On disait qu'il recevait des visiteurs étranges et les lumières qu'on voyait à ses fenêtres n'avaient pas toujours la même couleur. (Lovecraft, L'Affaire de Charles Dexter Ward)

(25) Faire le tour des appartements et nous montrer la serre sur laquelle s'ouvrait la dernière pièce et dont on disait des merveilles. (Gautier, Spirit)

On suggère un ensemble indéterminé de gens grâce aux implications du prédicat dire qui réalise le modèle sémantique : un humain dit quelque chose (proposition) à un autre humain, tandis que le morphème imperfectif ajouté au verbe dire, perfectif ajoute l'information sur l'itération de l'acte de dire. Il s'agit d'une série d'actes sur le même thème et le même rhème, mais avec plusieurs énonciateurs : l'information transmise reste la même, même si elle est reprise par plusieurs personnes. Cette itérativité du même propos lui ajoute une certaine pertinence, une idée de répétition d'une opinion, donc d'un propos communément et suffisamment accepté pour être connu d'un large public. 
En polonais, les constructions on $+V$, où le verbe est un marqueur évidentiel, sont rendues encore une fois par des constructions impersonnelles en - no ajouté à la variante imperfective du verbe: powiedzieć vs mówić. Comme c'est le cas du français, mówiono, mówi się, opowiadano, powiadano indiquent que la source du savoir est externe au locuteur et, comme en français, que l'iterativité est son mode d'action.

En voici quelques exemples :

(26) Przypadkowo znajdowatem się właśnie obok wymownego filozofa, o którym mówiono, że pogrąża się w uciechach światowych równie jak $i$ w teoriach kosmicznych. Mtoda kobieta, wychylając spoza firanek delikatna główkę i mała raczkę. (France, Pan Pigeaonneau, Jachimecka)

(27) Mówiono nawet, że to ona kazata przed wyjazdem spalić wszystkie meble. (Balzac, Tajemniczy dwór, trad. Krajewska)

(28) Och, to nie była pierwsza z zabaw nocnych, wydawanych co sezon przez nieobecnego lorda. I mówiono o tych festynach szeroko, gdyz ich ponura ekscentryczność graniczyła z fantastycznościa wobec niebrania w nich udziatu przez księcia. (Villiers de l'Isle-Adam, Duke of Portand, trad. Rogowicz)

Dans les exemples ci-dessous, la construction on $+V$ a été rendue par le verbe opowiadać ('raconter') qui dans (29) a été traduit par opowiadaja ('ils racontent') :

(29) Pan Cherbonneau ulegt zapewne wyczerpaniu wskutek nadmiernej pracy umystowej lub tė zginą przy jakimś śmialym doświadczeniu. Opowiadaja, że własnoręczny testament zmarlego, znaleziony $w$ biurku doktora, oddaje bibliotece Mazariniego niestychanie cenne rękopis. (Gautier, Avatar, trad. Parandowski)

(30) Widzicie więc, że pan Baltazar Cherbonneau byt najparadoksalniejszym doktorem na świecie i że przywiózł z Indii wyjątkowo ekscentryczne usposobienie; lecz stawa jego jako magnetyzera była jeszcze większa od stawy lekarza; $w$ gronie nielicznych wybranych urzqdzit kilka seansów, o których opowiadano cuda, zdolne pomieszać pojęcie możliwości i niemożliwości rzeczy, przewyższające wszystko, co czynit Cagliostro. (Gautier, Avatar, trad. Parandowski)

\section{CONCLUSION}

Notre objectif n'était pas une analyse de la valeur aspectuelle et de la valeur temporelle de la structure on $+V$. A cette étape, il s'agit seulement de repérer des emplois qui pourraient être significatifs dans le cadre des textes fantastiques. L'emploi des deux constructions analysées semble être intentionnel dans ce type de texte. Même si les deux structures renvoient aux situations différentes, le recours à la construction on $+V$ aboutit à une référence brouillée et perturbante. Atlani achève son étude sur le caractère "indécidable » du pronom on, et le présente comme 
" marque frontière entre la personne et la non-personne ", " entre ce qui est identifiable et donc nommable et ce qui ne l'est pas » (1984, p. 27).

Comme toute production, le fantastique résulte d'un certain nombre de pratiques, non pas ésotériques, mais bel et bien réelles et repérables. Il suffit de mettre le texte sous le microscope pour découvrir ces stratégies d'écriture. [...] à mettre au jour quelques-uns des procédés d'écriture qui concourent à l'établissement d'une vision fantastique (Morin, 1993, p. 132-133). «Le fantastique est une manière de raconter » (Bellemin-Noël, 1972, p. 3). Les personnages des contes fantastiques sont confrontés à l'innommable et l'immontrable. Le trait commun des constructions analysées est leur mouvance dans un réseau d'indéfinition et d'incertitude qui constitue un élément propre/normatif au fantastique. 


\section{BIBLIOGRAPHIE}

Atlani, F. (1984). « On l'illusionniste ». In : F. Atlani et al., La langue au ras du texte. Lille : Presses Universitaires de Lille, 13-29.

Balzac, H. (1975). « Tajemniczy dwór » (trad. Z. Krajewska). Fantastyczne opowieści. Antologia. Warszawa : Wydawnictwo Literackie.

Bellemin-Noël, J. (1972). « Le fantastique est une manière de raconter. Notes sur le Fantastique (textes de Théophile Gautier) ». Littérature, 8 (4), 3-23.

Blanche-Benveniste, C. (2003). « Le double jeu du pronom on ». In : M. Berre et al. (eds.), $L a$ syntaxe raisonnée. De Boeck Supérieur « Champs linguistiques », 41-56.

Bouvet, R. (2007). Etranges récits, étranges lectures. Essai sur l'effet fantastique. Québec: Presses de l'Université du Québec.

Charaudeau, P. (1992). Grammaire du sens et de l'expression. Paris : Hachette.

Dendale, P., Tasmowski, L. (1994). «Présentation. L'évidentialité ou le marquage des sources du savoir ». In : P. Dendale et L. Tasmowski (eds.). Langue française (102), Les sources du savoir et leurs marques linguistiques, 3-7.

France, A. (1975). «Pan Pigeonneau » (trad. Z. Jachimecka). Fantastyczne opowieści. Antologia. Warszawa : Wydawnictwo Literackie.

Gautier, T. (1980). Stopa mumii i inne opowiadania fantastyczne. Warszawa : Państwowy Instytut Wydawniczy.

Grevisse, M., Goosse, A. (2002). Le bon usage, $14^{\mathrm{e}}$ édition. Bruxelles : De Boeck Duculot.

Grzegorczykowa, R. (2008). Wstęp do językoznawstwa. Warszawa : PWN.

Karolak, S. (2000). « De la phrase impersonnelle au syntagme nominal ». Cahiers de l'ILSL, 12, 101-111.

Kim, M. (2004). « Une description des marqueurs évidentiels on dit que et on dirait que». Travaux de linguistique, 48, 41-52.

Krasnowolski, A. (1909). Systematyczna składnia języka polskiego. Warszawa: Wydawnictwo M. Arcta.

Landragin, F. (2007). «L'anaphore à antécédent flou : une caractérisation et ses conséquences sur l'annotation des relations anaphoriques ». Journée d'étude de l'Association pour le Traitement Automatique des Langues (ATALA) sur la résolution des anaphores. Jun 2007, Paris, France. Consulté le 10 octobre 2018 : https://halshs.archives-ouvertes.fr/halshs-00154860/document.

Landragin, F., Tanguy, N. (2014). «Référence et coréférence du pronom indéfini on ». Langages, 99-115.

Lecrivain, C. (1991). « Récit fantastique et traduction ». Actas del Primer Coloquio Internacional de Traductología, 137-138.

L'Isle-Adam, V. (1975a). «Duke of Portland» (trad. W. Rogowicz). Fantastyczne opowieści. Antologia. Warszawa : Wydawnictwo Literackie.

L'Isle-Adam, V. (1975b). «Wera» (trad. W. Rogowicz). Fantastyczne opowieści. Antologia. Warszawa : Wydawnictwo Literackie.

Mérimée, P. (1975a). «Lokis » (tard. Z. Jachimecka). Fantastyczne opowieści. Antologia. Warszawa : Wydawnictwo Literackie.

Mérimée, P. (1975b). «Wenus z Ille » (trad. Z. Jachimecka). Fantastyczne opowieści. Antologia. Warszawa : Wydawnictwo Literackie.

Miladi, L. (2012). Éléments du système linguistique de la langue polonaise : études contrastives (mémoire de synthèse).

Morin, L. (1993). «Quelques rouages de la machine fantastique dans les messagers de l'ascenseur ». Voix et Images, 19 (1), 132-150. 
Muryn, T. (2010). «Les constructions du type maintenant, aujourd'hui, à présent + imparfait comme expression du changement d'état dans un texte au passé ». In: J. Górnikiewicz, H. Grzmil-Tylutki, I. Piechnik (eds.), En quête de sens. Études dédiées à Marcela Świątkowska. Kraków : Wydawnictwo Uniwersytetu Jagiellońskiego, 347-353.

Muryn, T. (2019). «Rougir / devenir rouge : l'expression linguistique du langage corporel et ce qu'elle laisse inférer dans un genre textuel » (à paraître).

Netteberg, K. (1953). Études sur le verbe polonais. Copenhague : Rosenkilde og bagger.

Remi-Giraud, S. (2010). "De la matière à la forme : la déflexivité ou la naissance du mot». Langages, 2 (178), 53-66.

Sandfeld - Jensen, K. (1970). Syntaxe du français contemporain. I. Les pronoms. Paris : Honoré Champion.

Thuillier, F. (2004). " Le verbe paraître : surgissement,manifestation, impression, ouï-dire ». Linx 50.

Todorov, T. (1970). Introduction à la littérature fantastique. Paris : Seuil.

Weinrich, H. (1989). Grammaire textuelle du français. Paris : Les Éditions Didier.

Wolińska, O. (1978). Konstrukcje bezmianownikowe we współczesnej polszczyźnie. Katowice : Uniwersytet Śląski. 